\title{
CONNECTEDNESS OF PLANAR SELF-AFFINE SETS ASSOCIATED WITH NON-COLLINEAR DIGIT SETS
}

\author{
KING-SHUN LEUNG AND JUN JASON LUO
}

\begin{abstract}
We study the connectedness of the planar self-affine sets $T(A, \mathcal{D})$ generated by an integer expanding matrix $A$ with $|\operatorname{det}(A)|=3$ and a non-collinear digit set $\mathcal{D}=\{0, v, k A v\}$ where $k \in \mathbb{Z} \backslash\{0\}$ and $v \in \mathbb{Z}^{2}$ such that $\{v, A v\}$ is linearly independent. By checking the characteristic polynomials of $A$ case by case, we obtain a criterion concerning only $k$ to determine the connectedness of $T(A, \mathcal{D})$.
\end{abstract}

\section{Introduction}

Let $M_{n}(\mathbb{Z})$ denote the set of $n \times n$ matrices with integer entries, let $A \in M_{n}(\mathbb{Z})$ be expanding, i.e., all eigenvalues of $A$ have moduli strictly larger than 1. Assume $|\operatorname{det} A|=q$, and a finite set $\mathcal{D}=\left\{d_{1}, \ldots, d_{q}\right\} \subset \mathbb{R}^{n}$ with cardinality $q$, we call it a $q$-digit set. It is well known that there exists a unique self-affine set $T:=T(A, \mathcal{D})$ [12] satisfying:

$$
T=A^{-1}(T+\mathcal{D})=\left\{\sum_{i=1}^{\infty} A^{-i} d_{j_{i}}: d_{j_{i}} \in \mathcal{D}\right\} .
$$

$T$ is called a self-affine tile if such $T$ has a nonvoid interior.

The topological structure of $T(A, \mathcal{D})$, especially its connectedness, has attracted a lot of attentions in fractal geometry and number theory. It was asked by Gröchenig and Haas [5] that given an expanding integer matrix $A \in M_{n}(\mathbb{Z})$, whether there exists a digit set $\mathcal{D}$ such that $T(A, \mathcal{D})$ is a connected tile and they partially solved the question in $\mathbb{R}^{2}$. Hacon et al. [6] proved that any self-affine tile $T(A, \mathcal{D})$ with a 2-digit set is always pathwise connected. Lau and his collaborators ([8], [10], [11], [13]) systematically studied connectedness of the self-affine tiles generated by a kind of special digit sets of the form $\{0,1, \ldots, q-1\} v$ where $v \in \mathbb{Z}^{n} \backslash\{0\}$, which are called consecutive collinear digit sets. They observed a height reducing property (HRP) of the characteristic polynomial of $A$ to determine the connectedness of $T(A, \mathcal{D})$, and conjectured that all monic expanding polynomials have the HRP, thus all the tiles generated by consecutive collinear digit sets are connected. Akiyama and Gjini [1] solved it up to degree 4. However it is still open for arbitrary degree. In the plane,

Date: November 4, 2018.

Key words and phrases. connectedness, self-affine set, digit set, $\mathcal{E}$-connected.

The research is supported by STU Scientific Research Foundation for Talents. 
the disk-likeness (i.e. homeomorphic to a closed unit disk) is an interesting topic, Bandt and Gelbrich [2], Bandt and Wang [3], and Leung and Lau [13] investigated the disk-likeness of self-affine tiles in terms of the neighborhoods of $T$. Deng and Lau [4, and Kirat [9] concerned themselves about a class of planar self-affine tiles generated by product digit sets.

With regard to other types of digit sets, there are few results about the connectedness of $T(A, \mathcal{D})$ generated by non-consecutive or non-collinear digit sets. In [14], by counting the neighborhoods of $T$, the authors made a first attempt to exploit the case of non-consecutive collinear digit sets with $|\operatorname{det}(A)|=3$, and obtained a complete characterization for $T(A, \mathcal{D})$ to be connected or not. As a subsequent research, in the present paper, we will focus on the non-collinear digit sets. Precisely, we discuss the self-affine sets $T(A, \mathcal{D})$ generated by an expanding matrix $A$ with $|\operatorname{det}(A)|=3$ and a non-collinear digit set $\mathcal{D}=\{0, v, k A v\}$ for $k \in \mathbb{Z} \backslash\{0\}$ and $v \in \mathbb{R}^{2}$ such that $\{v, A v\}$ is linearly independent. By checking the characteristic polynomials of $A$ case by case, we obtain a criterion concerning only $k$ to determine the connectedness of $T(A, \mathcal{D})$.

Theorem 1.1. Let $A$ be a $2 \times 2$ integral expanding matrix with $|\operatorname{det}(A)|=3$, and let $\mathcal{D}=\{0, v, k A v\}$ be a digit set where $k \in \mathbb{Z} \backslash\{0\}$ such that $\{v, A v\}$ is linearly independent. Then the self-affine set $T(A, \mathcal{D})$ is connected if and only if $k= \pm 1$.

\section{Preliminaries}

In this section, we give some preparatory results of self-affine sets which will be used frequently in the paper. Define

$$
\mathcal{E}=\left\{\left(d_{i}, d_{j}\right):\left(T+d_{i}\right) \cap\left(T+d_{j}\right) \neq \emptyset, d_{i}, d_{j} \in \mathcal{D}\right\} .
$$

We say that $d_{i}$ and $d_{j}$ are $\mathcal{E}$-connected if there exists a finite sequence $\left\{d_{j_{1}}, \ldots, d_{j_{k}}\right\} \subset$ $\mathcal{D}$ such that $d_{i}=d_{j_{1}}, d_{j}=d_{j_{k}}$ and $\left(d_{j_{l}}, d_{j_{l+1}}\right) \in \mathcal{E}, 1 \leq l \leq k-1$.

It is easy to check that $\left(d_{i}, d_{j}\right) \in \mathcal{E}$ if and only if

$$
d_{i}-d_{j}=\sum_{k=1}^{\infty} A^{-k} v_{k} \quad \text { where } \quad v_{k} \in \Delta \mathcal{D}:=\mathcal{D}-\mathcal{D} .
$$

Then we get the following criterion of connectedness of a self-affine set.

Proposition 2.1. (17], [10]) A self-affine set $T$ with a digit set $\mathcal{D}$ is connected if and only if any two $d_{i}, d_{j} \in \mathcal{D}$ are $\mathcal{E}$-connected.

In the following, we mainly consider the planar self-affine set $T(A, \mathcal{D})$ generated by a $2 \times 2$ integral expanding matrix $A$ with $|\operatorname{det} A|=3$ and a digit set $\mathcal{D}=$ 
$\{0, v, k A v\}$ such that $\{v, A v\}$ is linearly independent, where $k \in \mathbb{Z} \backslash\{0\}$. Denote the characteristic polynomial of $A$ by $f(x)=x^{2}+p x+q$. Define $\alpha_{i}, \beta_{i}$ by

$$
A^{-i} v=\alpha_{i} v+\beta_{i} A v, \quad i=1,2, \ldots
$$

Applying the Hamilton-Cayley theorem $f(A)=A^{2}+p A+q I=0$, it follows a lemma.

Lemma 2.2. ([13]) Let $\alpha_{i}, \beta_{i}$ be defined as the above. Then $q \alpha_{i+2}+p \alpha_{i+1}+\alpha_{i}=0$ and $q \beta_{i+2}+p \beta_{i+1}+\beta_{i}=0$. Especially, $\alpha_{1}=-p / q, \alpha_{2}=\left(p^{2}-q\right) / q^{2} ; \beta_{1}=$ $-1 / q, \beta_{2}=p / q^{2}$. Moreover for $\Delta=p^{2}-4 q \neq 0$, we have

$$
\alpha_{i}=\frac{q\left(y_{1}^{i+1}-y_{2}^{i+1}\right)}{\Delta^{1 / 2}} \quad \text { and } \quad \beta_{i}=\frac{-\left(y_{1}^{i}-y_{2}^{i}\right)}{\Delta^{1 / 2}}
$$

where $y_{1}=\frac{-p+\Delta^{1 / 2}}{2 q}, y_{2}=\frac{-p-\Delta^{1 / 2}}{2 q}$ are the two roots of $q x^{2}+p x+1=0$.

Let

$$
\tilde{\alpha}:=\sum_{i=1}^{\infty}\left|\alpha_{i}\right|, \quad \tilde{\beta}:=\sum_{i=1}^{\infty}\left|\beta_{i}\right| .
$$

Corollary 2.3. Assume $f(x)=x^{2}+p x+q$ and $g(x)=x^{2}-p x+q$ be the characteristic polynomials of expanding matrices $A$ and $B$, respectively. Let $\alpha_{i}, \beta_{i}, \tilde{\alpha}, \tilde{\beta}$ for $f(x)$ be as before; let $\alpha_{i}^{\prime}, \beta_{i}^{\prime}, \tilde{\alpha}^{\prime}, \tilde{\beta}^{\prime}$ be the corresponding terms for $g(x)$. Then

$$
\alpha_{2 j}^{\prime}=\alpha_{2 j}, \quad \alpha_{2 j-1}^{\prime}=-\alpha_{2 j-1}, \beta_{2 j}^{\prime}=-\beta_{2 j}, \beta_{2 j-1}^{\prime}=\beta_{2 j-1},
$$

and hence $\tilde{\alpha}=\tilde{\alpha^{\prime}}, \tilde{\beta}=\tilde{\beta}^{\prime}$.

When $|\operatorname{det} A|=3$, it is known by [2] that there are 10 eligible characteristic polynomials of $A$ :

$$
x^{2} \pm 3 ; \quad x^{2} \pm x+3 ; \quad x^{2} \pm 2 x+3 ; \quad x^{2} \pm 3 x+3 ; \quad x^{2} \pm x-3 .
$$

Following [14], together with Corollary 2.3, we obtained the estimates or values of the corresponding $\tilde{\alpha}$ and $\tilde{\beta}$ as follows:

$$
\begin{array}{ll}
f(x)=x^{2} \pm x+3: & \tilde{\alpha}<0.88, \tilde{\beta}<0.63 \\
f(x)=x^{2} \pm 2 x+3: & \tilde{\alpha}<1.17, \tilde{\beta}<0.73 \\
f(x)=x^{2} \pm 3 x+3: & \tilde{\alpha}<2.24, \tilde{\beta}<1.08 \\
f(x)=x^{2} \pm x-3: & \tilde{\alpha}=2, \tilde{\beta}=1 .
\end{array}
$$




\section{Main results}

For a digit set $\mathcal{D}=\{0, v, k A v\}$, we denote by $\Delta \mathcal{D}=\{0, \pm v, \pm(k A v-v), \pm k A v\}$ the difference set. First we show the following simplest case according to the characteristic polynomials of $A$.

Theorem 3.1. Let $A$ be $a 2 \times 2$ integral expanding matrix with characteristic polynomial $f(x)=x^{2} \pm 3$ and $\mathcal{D}=\{0, v, k A v\}$ be a digit set where $k \in \mathbb{Z} \backslash\{0\}$ such that $\{v, A v\}$ is linearly independent. Then the self-affine set $T(A, \mathcal{D})$ is connected if and only if $k= \pm 1$.

Proof. Since the case of $f(x)=x^{2}-3$ is more or less the same as that of $f(x)=x^{2}+3$, it suffices to show the last one. If $k=1$, then $\Delta \mathcal{D}=\{0, \pm v, \pm(A v-v), \pm A v\}$. From $f(A)=A^{2}+3 I=0$, we have

$$
I=-2 A^{-2}\left(I+A^{-2}\right)^{-1}=2 \sum_{n=1}^{\infty}(-1)^{n} A^{-2 n}
$$

and

$$
v=2 \sum_{n=1}^{\infty}(-1)^{n} A^{-2 n} v=\sum_{n=0}^{\infty} A^{-4 n}\left(A^{-2}(-v)+A^{-3}(-A v)+A^{-4} v+A^{-5}(A v)\right)
$$

Hence $v \in T-T$, or equivalently $T \cap(T+v) \neq \emptyset$. Moreover,

$$
A v=\sum_{n=0}^{\infty} A^{-4 n}\left(A^{-1}(-v)+A^{-2}(-A v)+A^{-3} v+A^{-4}(A v)\right)
$$

which implies $T \cap(T+A v) \neq \emptyset$. Consequently, by Proposition 2.1. $T$ is connected (see Figure 1(a)).

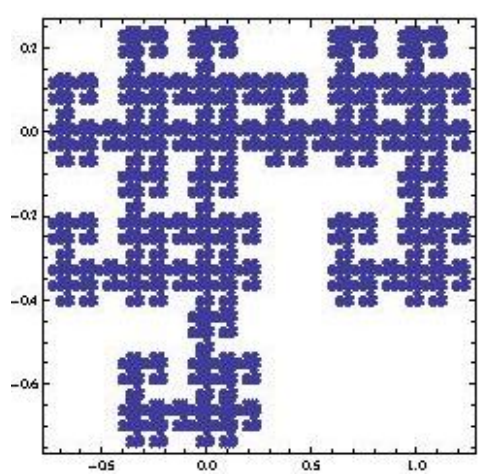

(a) $k=1$

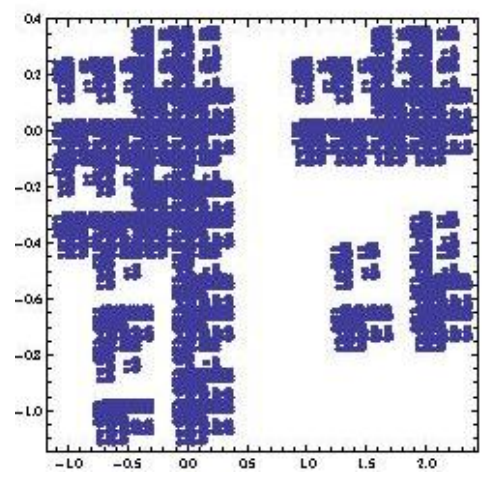

(b) $k=2$

Figure 1. Two cases for $f(x)=x^{2}+3$ and $v=(1,0)^{t}$.

If $k=-1$, then $\Delta \mathcal{D}=\{0, \pm v, \pm(A v+v), \pm A v\}$, and (3.1), (3.2), (3.3) still hold. Hence $T$ is also connected. 
If $|k|>1$, let $k_{i} A v+l_{i} v \in \Delta \mathcal{D}$ for $i \geq 1$, then a point of $T-T$ can be written as

$$
\begin{aligned}
& \sum_{i=1}^{\infty} A^{-i}\left(k_{i} A v+l_{i} v\right)=\sum_{i=1}^{\infty} A^{-2 i}\left(k_{2 i} A v+l_{2 i} v\right)+\sum_{i=1}^{\infty} A^{-2 i+1}\left(k_{2 i-1} A v+l_{2 i-1} v\right) \\
= & \sum_{i=1}^{\infty}\left(-\frac{1}{3}\right)^{i}\left(k_{2 i} A v+l_{2 i} v\right)+\sum_{i=1}^{\infty}\left(-\frac{1}{3}\right)^{i}\left(-3 k_{2 i-1} v+l_{2 i-1} A v\right) \\
= & \left(k_{1}+\sum_{i=1}^{\infty}\left(-\frac{1}{3}\right)^{i}\left(l_{2 i}+k_{2 i+1}\right)\right) v+\left(\sum_{i=1}^{\infty}\left(-\frac{1}{3}\right)^{i}\left(l_{2 i-1}+k_{2 i}\right)\right) A v \\
:= & L v+K A v .
\end{aligned}
$$

As $\left|l_{i}+k_{i+1}\right| \leq 1+|k|$, it follows that $|K| \leq(1+|k|) \sum_{i=1}^{\infty}\left(\frac{1}{3}\right)^{i}=(1+|k|) / 2<|k|$. Hence $T \cap(T+k A v)=\emptyset$ and $(T+v) \cap(T+k A v)=\emptyset$, which imply that $T$ is disconnected (see Figure $1(\mathrm{~b}))$.

Theorem 3.2. Let $A$ be a $2 \times 2$ integral expanding matrix with characteristic polynomial $f(x)=x^{2}+p x \pm 3$ where $p>0$, and let $\mathcal{D}=\{0, v, k A v\}$ be a digit set where $k \in \mathbb{Z} \backslash\{0\}$ such that $\{v, A v\}$ is linearly independent. Then the self-affine set $T(A, \mathcal{D})$ is connected if and only if $k= \pm 1$.

Proof. For the cases of $f(x)=x^{2}+p x+3$ with $0<p<3$, by using $0=f(A)=$ $f(A)(A-I)=A^{3}+(p-1) A^{2}+(3-p) A-3 I$, we obtain

$$
I=\sum_{i=1}^{\infty} A^{-3 i}\left((1-p) A^{2}-(3-p) A+2 I\right)
$$

Case 1. $f(x)=x^{2}+x+3$ : For $k=1$, then $\Delta \mathcal{D}=\{0, \pm v, \pm(A v-v), \pm A v\}$. By (3.4), $I=\sum_{i=1}^{\infty} A^{-3 i}(-2 A+2 I)$ and

$$
v=\sum_{i=1}^{\infty} A^{-3 i}(-2 A v+2 v)=\sum_{i=0}^{\infty} A^{-3 i}\left(A^{-2}(-v)+A^{-3}(v-A v)+A^{-4}(A v)\right)
$$

Hence $T \cap(T+v) \neq \emptyset$. Moreover,

$$
A v=\sum_{i=0}^{\infty} A^{-3 i}\left(A^{-1}(-v)+A^{-2}(v-A v)+A^{-3}(A v)\right)
$$

which implies $T \cap(T+A v) \neq \emptyset$. Consequently, $T$ is connected (see Figure $2(\mathrm{a})$ ). 


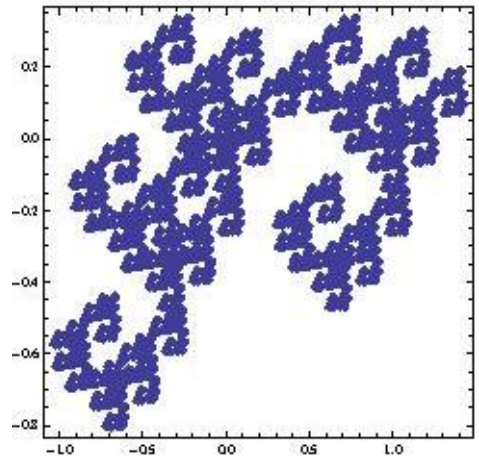

(a) $x^{2}+x+3$

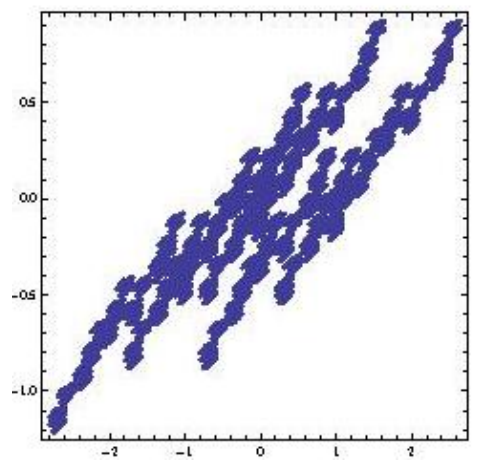

(c) $x^{2}+3 x+3$

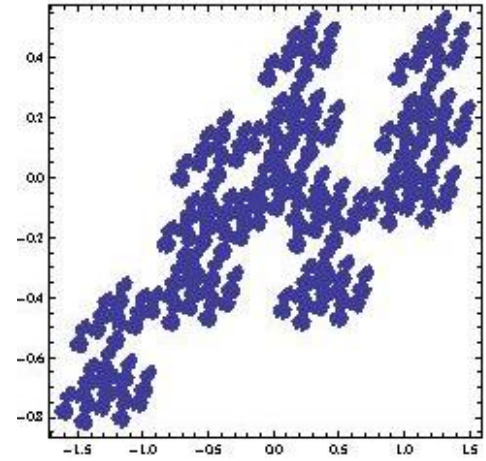

(b) $x^{2}+2 x+3$

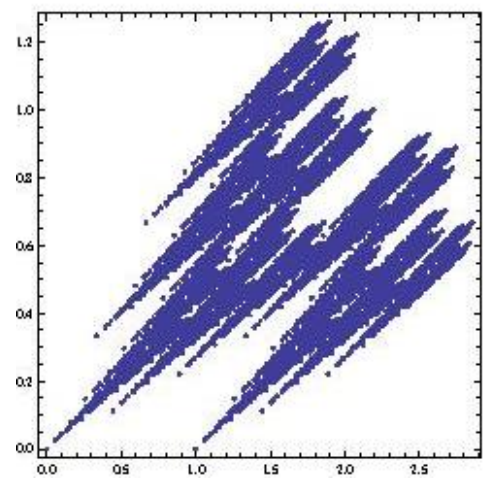

(d) $x^{2}+x-3$

Figure 2. Connected cases for $v=(1,0)^{t}$ and $k=1$.

For $k=-1$, then $\Delta \mathcal{D}=\{0, \pm v, \pm(A v+v), \pm A v\}$. From $f(A)=0$, we deduce that $I=(-A-2 I)\left(A^{2}+I\right)^{-1}$, which in turn gives

$$
\begin{aligned}
v= & -A^{-1} v-2 A^{-2} v+A^{-3} v+2 A^{-4} v-A^{-5} v-2 A^{-6} v+A^{-7} v+2 A^{-8} v-\cdots \\
= & A^{-2}(-A v-v)+A^{-3}(-A v)+A^{-4}(A v+v)+A^{-5}(A v)+A^{-6}(-A v-v) \\
& +A^{-7}(-A v)+A^{-8}(A v+v)+A^{-9}(A v)+\cdots \\
\in & T-T .
\end{aligned}
$$

Hence $T \cap(T+v) \neq \emptyset$. Multiplying the above expression by $A$, we have

$$
\begin{aligned}
A v= & A^{-1}(-A v-v)+A^{-2}(-A v)+A^{-3}(A v+v)+A^{-4}(A v) \\
& +A^{-5}(-A v-v)+A^{-6}(-A v)+A^{-7}(A v+v)+A^{-8}(A v)+\cdots \\
\in & T-T
\end{aligned}
$$

which implies $T \cap(T+A v) \neq \emptyset$. It follows that $T$ is connected. 
For $|k|>1$. A point of $T-T$ can be written as

$$
\sum_{i=1}^{\infty} A^{-i}\left(k_{i} A v+l_{i} v\right)
$$

where $k_{i} A v+l_{i} v \in \Delta \mathcal{D}$ for $i \geq 1$. By using the relation $A^{-i} v=\alpha_{i} v+\beta_{i} A v$,

$$
\begin{aligned}
& \sum_{i=1}^{\infty} A^{-i}\left(k_{i} A v+l_{i} v\right)=\sum_{i=1}^{\infty}\left(k_{i} A^{-i+1} v+l_{i} A^{-i} v\right) \\
= & \sum_{i=1}^{\infty} k_{i}\left(\alpha_{i-1} v+\beta_{i-1} A v\right)+\sum_{i=1}^{\infty} l_{i}\left(\alpha_{i} v+\beta_{i} A v\right) \\
= & \left(k_{1}+\sum_{i=1}^{\infty}\left(k_{i+1}+l_{i}\right) \alpha_{i}\right) v+\left(\sum_{i=1}^{\infty}\left(k_{i+1}+l_{i}\right) \beta_{i}\right) A v \\
:= & L v+K A v .
\end{aligned}
$$

As $\left|l_{i}+k_{i+1}\right| \leq 1+|k|$ and $\tilde{\beta}<0.63(2.1)$, we conclude $|K| \leq 0.63(1+|k|)<|k|$, which yields $T \cap(T+k A v)=\emptyset$ and $(T+v) \cap(T+k A v)=\emptyset$. Hence $T$ is disconnected (see Figure 3(a)).

Case 2. $f(x)=x^{2}+2 x+3$ : For $k=1$. By (3.4), $I=\sum_{i=1}^{\infty} A^{-3 i}\left(-A^{2}-A+2 I\right)$ and

$v=\sum_{i=1}^{\infty} A^{-3 i}\left(-A^{2} v-A v+2 v\right)=A^{-1}(-v)+\sum_{i=0}^{\infty} A^{-3 i}\left(A^{-2}(-v)+A^{-3} v+A^{-4}(A v-v)\right)$.

Hence $T \cap(T+v) \neq \emptyset$. Moreover,

$$
A v=A^{-1}(-A v)+\sum_{i=0}^{\infty} A^{-3 i}\left(A^{-1}(-v)+A^{-2} v+A^{-3}(A v-v)\right)
$$

which implies $T \cap(T+A v) \neq \emptyset$. Consequently, $T$ is connected (see Figure 2(b)).

For $k=-1$. We obtain from (3.4) that

$$
\begin{aligned}
v= & -A^{-1} v-A^{-2} v+2 A^{-3} v-A^{-4} v-A^{-5} v \\
& +2 A^{-6} v-A^{-7} v-A^{-8} v+2 A^{-9} v+\cdots \\
= & A^{-1}(-v)+A^{-2}(-v)+A^{-3} v+A^{-4}(A v)+A^{-5}(-A v-v) \\
& +A^{-6} v+A^{-7}(A v)+A^{-8}(-A v-v)+\cdots \\
\in & T-T,
\end{aligned}
$$

implying $T \cap(T+v) \neq \emptyset$. Multiplying the above expression by $A$, we have

$$
\begin{aligned}
A v+v= & A^{-1}(-v)+A^{-2} v+A^{-3}(A v)+A^{-4}(-A v-v) \\
& +A^{-5} v+A^{-6}(A v)+A^{-7}(-A v-v)+\cdots \\
\in & T-T,
\end{aligned}
$$

implying $(T+v) \cap(T-A v) \neq \emptyset$. Hence $T$ is connected. 


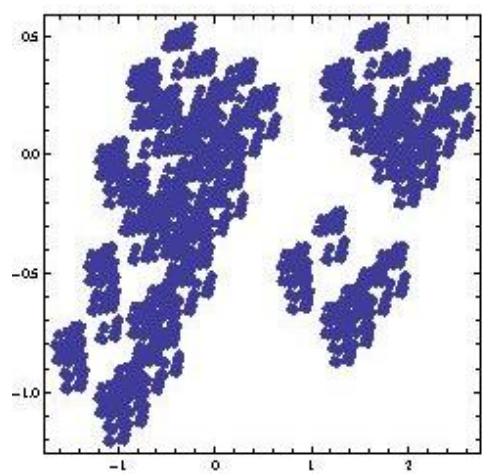

(a) $x^{2}+x+3$

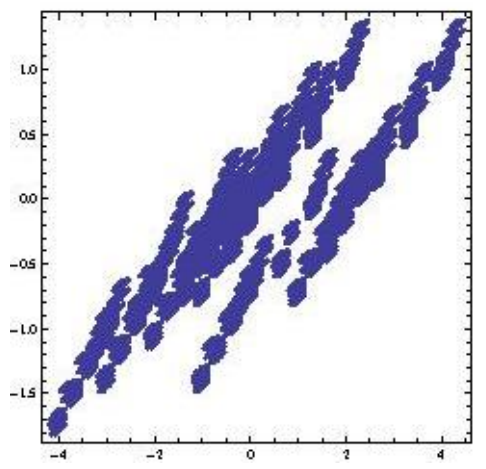

(c) $x^{2}+3 x+3$

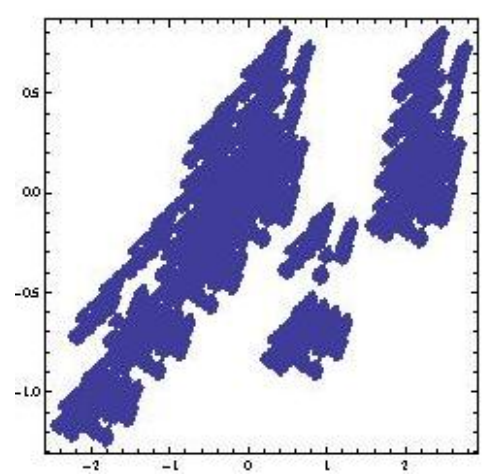

(b) $x^{2}+2 x+3$

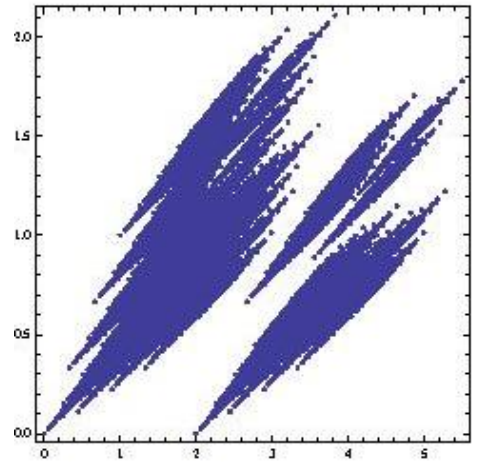

(d) $x^{2}+x-3$

Figure 3. Disconnected cases for $v=(1,0)^{t}$ and $k=2$.

For $|k|>1$. By 3.7 and $\tilde{\beta}<0.73 \sqrt{2.2}$, we have $|K| \leq(1+|k|) \tilde{\beta}<0.73(1+$ $|k|)$. When $|k| \geq 3,|K|<0.73(1+|k|)<|k|$, which yields $T \cap(T+k A v)=\emptyset$ and $(T+v) \cap(T+k A v)=\emptyset$. Hence $T$ is disconnected. When $k=2$, suppose $(T+2 A v) \cap T \neq \emptyset$ or $(T+2 A v) \cap(T+v) \neq \emptyset$, i.e., $2 A v+l v \in T-T$ for $l=0$ or -1 . By (3.7), we obtain

$$
\left(k_{2}+l_{1}\right) \beta_{1}=2-\sum_{i=2}^{\infty}\left(k_{i+1}+l_{i}\right) \beta_{i} \geq 2-(1+2)\left(\tilde{\beta}-\left|\beta_{1}\right|\right)>0.8
$$

where $\beta_{1}=-1 / 3$. It follows that $l_{1}=-1, k_{2}=-2$. Then using $f(A)=0$, we have

$$
A(2 A v+l v)-k_{1} A v-l_{1} v=\left(-4+l-k_{1}\right) A v-\left(6+l_{1}\right) v \in T-T .
$$

It follows from $l_{1}=-1$ that $k_{1}=0$ or 2 . Hence $-4+l-k_{1} \leq-4+0+0=-4$, which contradicts the inequality $|K| \leq(1+|k|) \tilde{\beta}<3 \times 0.73=2.19$. So $T$ is disconnected for $k=2$ (see Figure $3(\mathrm{~b})$ ).

When $k=-2$, suppose $2 A v+l v \in T-T$ where $l=0$ or 1 . Similarly, it yields from (3.8) that $l_{1}=-1, k_{2}=-2$ and $k_{1}=-2$ or 0 . If $k_{1}=-2$, then 
$(l-2) A v-5 v \in T-T$. Multiplying the expression by $A$ and using $f(A)=0$, we obtain

$$
(l-2) A^{2} v-5 A v-\left(k_{2} A v+l_{2} v\right)=(1-2 l) A v+\left(6-3 l-l_{2}\right) v \in T-T
$$

and $6-3 l-l_{2} \geq 6-3-1=2$. On the other hand, by (3.7) and (2.2), $-5.81=-2-$ $3 \tilde{\alpha} \leq L \leq-2+3 \tilde{\alpha}<1.81$. This is ridiculous. If $k_{1}=0$, then $(l-4) A v-5 v \in T-T$ and $|l-4| \geq 3$ contracts $|l-4|=|K|<2.19$. Therefore $T$ is disconnected for $k=-2$.

Case 3. $f(x)=x^{2}+3 x+3$ : For $k=1$. From $(A-I) f(A)=0$, we get $A^{2}+A-I=2(A+I)^{-1}$, which yields $I=-A^{-1}+A^{-2}+2 \sum_{i=3}^{\infty}(-1)^{i+1} A^{-i}$ and

$$
\begin{aligned}
v & =-A^{-1} v+A^{-2} v+2 \sum_{i=3}^{\infty}(-1)^{i+1} A^{-i} v \\
& =A^{-2}(v-A v)+A^{-3} v+\sum_{i=0}^{\infty} A^{-2 i}\left(A^{-4}(A v-v)+A^{-5}(v-A v)\right) .
\end{aligned}
$$

Hence $T \cap(T+v) \neq \emptyset$. Moreover,

$$
A v=A^{-1}(v-A v)+A^{-2} v+\sum_{i=0}^{\infty} A^{-2 i}\left(A^{-3}(A v-v)+A^{-4}(v-A v)\right)
$$

which implies $T \cap(T+A v) \neq \emptyset$. Consequently, $T$ is connected (see Figure 2(c)).

For $k=-1$. From $f(A)=0$ we get $A+2 I=-(A+I)^{-1}$. It follows that $I=-2 A^{-1}-A^{-2}+A^{-3}-A^{-4}+A^{-5}+\cdots$ and

$$
\begin{aligned}
v & =-2 A^{-1} v-A^{-2} v+A^{-3} v-A^{-4} v+A^{-5} v+\cdots \\
& =A^{-1}(-v)+A^{-2}(-A v-v)+A^{-3} v+A^{-4}(-v)+A^{-5} v+A^{-6}(-v)+\cdots \\
& \in T-T,
\end{aligned}
$$

then $T \cap(T+v) \neq \emptyset$. Also we can deduce immediately that

$$
\begin{aligned}
A v+v & =A^{-1}(-A v-v)+A^{-2} v+A^{-3}(-v)+A^{-4} v+A^{-5}(-v)+\cdots \\
& \in T-T
\end{aligned}
$$

which yields $(T+v) \cap(T-A v) \neq \emptyset$. As a result, $T$ is connected.

For $k>1$. By (3.7) and (2.3), we have $|L| \leq k+(1+k) \tilde{\alpha}<2.24+3.24 k$. Suppose $k A v+l v \in T-T$ for $l=0$ or -1 . Multiplying (3.7) by $A$ and then subtracting $k_{1} A v+l_{1} v$ from both sides, we see that

$$
\left(-3 k+l-k_{1}\right) A v-\left(3 k+l_{1}\right) v \in T-T .
$$

Repeating the process, we obtain

$$
\left(6 k-3 l+3 k_{1}-l_{1}-k_{2}\right) A v+\underset{9}{\left(9 k-3 l+3 k_{1}-l_{2}\right) v \in T-T .}
$$


Since $9 k-3 l+3 k_{1}-l_{2} \geq 9 k-0-3 k-1=6 k-1>2.24+3.24 k$, which exceeds the upper bound of $|L|$. It concludes that $T \cap(T+k A v)=\emptyset$ and $(T+v) \cap(T+k A v)=\emptyset$, that is, $T$ is disconnected (see Figure $3(\mathrm{c})$ ).

For $k \leq-3$, then $|L| \leq-k+(1-k) \tilde{\alpha}<2.24-3.24 k$. It follows from (3.10) that $\left|9 k-3 l+3 k_{1}-l_{2}\right| \geq-9 k-3+3 k-1=-6 k-4>2.24-3.24 k$, which also exceeds the upper bound of $|L|$.

For $k=-2$, then $|K|<3.24$ and $|L|<8.72$. From (3.9), we have $6+l-k_{1}<3.24$, hence $l=-1$ and $k_{1}=2$. From $(3.10)$, we have $l_{2}=-1$ and $3+l_{1}+k_{2}<3.24$, it follows that $l_{1}=0, k_{2}=-2$, or $l_{1}=0, k_{2}=0$, or $l_{1}=1, k_{2}=-2$.

When $l_{1}=0, k_{2}=-2$. Multiplying (3.10) by $A$ and then subtracting $k_{3} A v+l_{3} v$, we get

$$
\left(-5-k_{3}\right) A v+\left(3-l_{3}\right) v \in T-T .
$$

By $\left|5+k_{3}\right| \leq 3.24$, it yields $k_{3}=-2$. Repeating this process, we obtain

$$
\left(12-l_{3}-k_{4}\right) A v+\left(9-l_{4}\right) v \in T-T .
$$

Hence we get a contradiction $\left|12-l_{3}-k_{4}\right| \geq 9>3.24$.

When $l_{1}=0, k_{2}=0$. Multiplying (3.10) by $A$ and then subtracting $k_{3} A v+l_{3} v$, we get

$$
\left(1-k_{3}\right) A v+\left(9-l_{3}\right) v \in T-T .
$$

It yields $l_{3}=1$ and $k_{3}=0$ or 2 . Repeating this process, we obtain

$$
\left(3 k_{3}+5-k_{4}\right) A v+\left(3 k_{3}-3-l_{4}\right) v \in T-T .
$$

If $k_{3}=0$, then $\left(5-k_{4}\right) A v+\left(-3-l_{4}\right) v \in T-T$, and $k_{4}=2$. Finally we get $\left(-12-l_{4}-k_{5}\right) A v+\left(-9-l_{5}\right) v \in T-T$ and a contradiction $\left|12+l_{4}+k_{5}\right| \geq 9>3.24$. If $k_{3}=2$, then $\left(11-k_{4}\right) A v+\left(3-l_{4}\right) v \in T-T$, and also $\left|11-k_{4}\right| \geq 9>3.24$.

When $l_{1}=1, k_{2}=-2$. By the same argument as above, we first get

$$
\left(-2-k_{3}\right) A v+\left(6-l_{3}\right) v \in T-T .
$$

It yields $k_{3}=0$. Repeating this process, we obtain $\left(12-l_{3}-k_{4}\right) A v+\left(6-l_{4}\right) v \in T-T$ and $\left|12-l_{3}-k_{4}\right| \geq 9>3.24$ follows. Therefore $T$ is disconnected for $|k|>1$.

Case 4. $f(x)=x^{2}+x-3$ : For $k=1$. We deduce from $f(A)=0$ that $I=\left(A^{2}-I\right)^{-1}(-A+2 I)$, which yields $I=\sum_{i=0}^{\infty} A^{-2 i}\left(-A^{-1}+2 A^{-2}\right)$ and

$$
\begin{aligned}
v & =\sum_{i=0}^{\infty} A^{-2 i}\left(-A^{-1}+2 A^{-2}\right) v \\
& =A^{-2}(v-A v)+\sum_{i=1}^{\infty} A^{-2 i}\left(A^{-1}(A v-v)+A^{-2} v\right) .
\end{aligned}
$$

Hence $T \cap(T+v) \neq \emptyset$. Moreover,

$$
A v=A^{-1}(v-A v)+\sum_{i=1}^{\infty} A^{-2 i}\left((A v-v)+A^{-1} v\right)
$$


which implies $T \cap(T+A v) \neq \emptyset$. Consequently, $T$ is connected (see Figure $2(\mathrm{~d})$ ).

For $k=-1$. It follows from $v=A^{-1}(A v) \in T-T$ that $T \cap(T+v) \neq \emptyset$. Moreover, we can get $A+I=-I+(A-I)^{-1}$ from $f(A)=0$. This implies

$$
\begin{aligned}
A v+v & =A^{-1}(-A v)+A^{-2}(A v)+A^{-3}(A v)+A^{-4}(A v)+\cdots \\
& \in T-T,
\end{aligned}
$$

that is, $(T+v) \cap(T-A v) \neq \emptyset$. Hence $T$ is connected.

For $k>1$. By $(3.7)$ and $(2.4)$, we have $|K| \leq(1+k) \tilde{\beta}=1+k$ and $|L| \leq$ $k+(1+k) \tilde{\alpha}=2+3 k$. Suppose $k A v+l v \in T-T$ for $l=0$ or -1 . Multiplying (3.7) by $A$ and then subtracting $k_{1} A v+l_{1} v$ from both sides, we have

$$
\left(-k+l-k_{1}\right) A v+\left(3 k-l_{1}\right) v \in T-T .
$$

Repeating the process, we obtain

$$
\left(4 k-l+k_{1}-l_{1}-k_{2}\right) A v+\left(-3 k+3 l-3 k_{1}-l_{2}\right) v \in T-T .
$$

Note $4 k-l+k_{1}-l_{1}-k_{2} \geq 2 k-1$. When $k \geq 3,2 k-1>k+1$ which contradicts the upper bound of $|K|$, hence $T$ is disconnected; when $k=2$, it forces $l=0, k_{1}=$ $-2, l_{1}=1, k_{2}=2$ and $3 A v-l_{2} v \in T-T$, similarly which implies

$$
\left(-3-l_{2}-k_{3}\right) A v+\left(9-l_{3}\right) v \in T-T .
$$

It is required that $\left|9-l_{3}\right| \leq 8$, hence $l_{3}=1$. Furthermore, from $\left(-3-l_{2}-k_{3}\right) A v+8 v \in$ $T-T$, we can deduce that

$$
\left(11+l_{2}+k_{3}-k_{4}\right) A v+\left(-9-3 l_{2}-3 k_{2}-l_{4}\right) v \in T-T .
$$

Since $11+l_{2}+k_{3}-k_{4} \geq 6>3$, we also get a contradiction, and $T$ is disconnected. Consequently, $T$ is disconnected for all $k>1$ (see Figure $3(\mathrm{~d})$ ).

For $k<-1$, then $|K| \leq 1-k$. From (3.11), it follows that $|K| \geq-4 k+l-k_{1}+$ $l_{1}+k_{2} \geq-4 k-1+k+1+k=-2 k>1-k$, which is impossible. Therefore $T$ is disconnected for $|k|>1$.

Theorem 3.3. Let $B \in M_{2}(\mathbb{Z})$ be an expanding integral matrix with characteristic polynomial $g(x)=x^{2}-p x \pm 3$ where $p>0$, and let $\mathcal{D}=\{0, v, k B v\}$ be a digit set where $k \in \mathbb{Z} \backslash\{0\}$ such that $\{v, B v\}$ is linearly independent. Then the self-affine set $T(B, \mathcal{D})$ is connected if and only if $k= \pm 1$.

Proof. The characteristic polynomial of $A$ is $f(x)=x^{2}+p x+q$ if and only if that of $-A$ is $g(x)=x^{2}-p x+q$. Since only the radix expansions matter, we may assume $B=-A$. The proof for the disconnectedness of $T(B, \mathcal{D})$ when $|k|>1$ can be adapted easily from the proof of Theorem 3.2 by applying Corollary 2.3. Let $\mathcal{D}=\{0, v, A v\}=\{0, v,-B v\}$ and $\mathcal{D}^{\prime}=\{0, v,-A v\}=\{0, v, B v\}$. For $|k|=1$, we deduce the connectedness of $T_{1}:=T(B, \mathcal{D})$ (respectively, $T_{1}^{\prime}=T\left(B, \mathcal{D}^{\prime}\right)$ ) from 
that of $T=T(A, \mathcal{D})$ (respectively, $T^{\prime}=T\left(A, \mathcal{D}^{\prime}\right)$ ). We only show the case of $f(x)=x^{2}+x+3$. In Case 1 of the proof of Theorem 3.2, from 3.5 we have

$$
v=\sum_{i=0}^{\infty}(-B)^{-3 i}\left(B^{-2}(-v)-B^{-3}(v+B v)+B^{-4}(-B v)\right) \in T_{1}-T_{1} ;
$$

and from 3.6$)$ we have

$$
B v=\sum_{i=0}^{\infty}(-B)^{-3 i}\left(B^{-1}(-v)-B^{-2}(v+B v)+B^{-3}(-B v)\right) \in T_{1}-T_{1} .
$$

Hence $T_{1}$ is connected. Similarly, it can be verified that $v, B v \in T_{1}^{\prime}-T_{1}^{\prime}$ and $T_{1}^{\prime}$ is also connected.

The above proof is indeed an application of the following more general result.

Theorem 3.4. Let $A, B \in M_{2}(\mathbb{Z})$ be two expanding integral matrices with characteristic polynomials $f(x)=x^{2}+p x+q$ and $g(x)=x^{2}-p x+q$ respectively. Let $v, w$ be two non-zero vectors such that the two sets $\{v, A v\}$ and $\{w, B w\}$ are both linearly independent. Denote by $L$ and $M$ the lattices generated by $\{v, A v\}$ and $\{w, B w\}$ respectively. Let $\mathcal{D}=\left\{c_{i} A v+d_{i} v \in L: i=0,1, \ldots,|q|-1\right\}$ and $\mathcal{D}^{\prime}=\left\{-c_{i} B w+d_{i} w \in M: i=0,1, \ldots,|q|-1\right\}$ be two digit sets. Then $T_{1}=T(A, \mathcal{D})$ is connected if and only if $T_{2}=T\left(B, \mathcal{D}^{\prime}\right)$ is connected.

Proof. We may assume $B=-A$ and $w=-v$. Then $\mathcal{D}=\mathcal{D}^{\prime}$ and $L=M$. Consider $a_{i} A v+b_{i} v=-a_{i} B v+b_{i} v \in \Delta \mathcal{D}=\Delta \mathcal{D}^{\prime}$, it follows that $a_{i} A v+b_{i} v=A^{-1}\left(c_{1}^{\prime} A v+\right.$ $\left.d_{1}^{\prime} v\right)+A^{-2}\left(c_{2}^{\prime} A v+d_{2}^{\prime} v\right)+\cdots \in T_{1}-T_{1}$ if and only if $-a_{i} B v+b_{i} v=B^{-1}\left(c_{1}^{\prime} B v-\right.$ $\left.d_{1}^{\prime} v\right)+B^{-2}\left(-c_{2}^{\prime} B v+d_{2}^{\prime} v\right)+\cdots \in T_{2}-T_{2}$. Hence $c_{i} A v+d_{i} v, c_{j} A v+d_{j} v \in \mathcal{D}$ are $\mathcal{E}$-connected if and only if $-c_{i} B v+d_{i} v,-c_{j} B v+d_{j} v \in \mathcal{D}^{\prime}$ are $\mathcal{E}$-connected, then the theorem is proved by Proposition 2.1.

We can also deduce from Theorems 3.2 and 3.3 that

Corollary 3.5. Let $A$ be a $2 \times 2$ integral expanding matrix with characteristic polynomial $f(x)=x^{2}+p x+q$ where $|q|=3$. Let $v \in \mathbb{R}^{2}$ such that $\{v, A v\}$ is linearly independent. Then the self-affine set $T(A, \mathcal{D})$ is connected for $\mathcal{D}=\{0, v, A v+v\}$ or $\{0, v,-A v+v\}$.

Proof. Notice that the difference set $\Delta\{0, v, A v+v\}=\{0, \pm v, \pm A v, \pm(A v+v)\}=$ $\Delta\{0, v,-A v\}$ and $\Delta\{0, v,-A v+v\}=\{0, \pm v, \pm A v, \pm(A v-v)\}=\Delta\{0, v, A v\}$. Hence the result follows from Proposition 2.1.

Remarks: The connectedness of self-affine sets or self-affine tiles is far from known extensively. Even for the planar case, there are still a lot of unsolved questions. The following may be some interesting topics related to the paper. 
Q1. Can we characterize the connectedness of $T(A, \mathcal{D})$ with $|\operatorname{det}(A)|=3$ and $\mathcal{D}=\{0, v, k A v+l v\} ?$

Q2. For a two dimensional digit set $\mathcal{D}=\{0, v, \ldots,(l-1) v, A v, \ldots, k A v\}$ with $l+k=|\operatorname{det}(A)|>3$, can we apply the same method to study the connectedness of $T(A, \mathcal{D}) ?$

Acknowledgments: The authors would like to thank Professor Ka-Sing Lau for suggesting a related question and advice on the work.

\section{REFERENCES}

[1] S. Akiyama and N. Gjini, Connectedness of number-theoretic tilings, Discrete Math. Theoret. Computer Science 7 (2005), no. 1, 269-312.

[2] C. Bandt and G. Gelbrich, Classification of self-affine lattice tilings, J. London Math. Soc. 50 (1994), 581-593.

[3] C. Bandt and Y. Wang, Disk-like self-affine tiles in $\mathbb{R}^{2}$, Discrete Comput. Geom. 26 (2001), no.4, 591-601.

[4] Q.R. Deng and K.S. Lau, Connectedness of a class of planar self-affine tiles, J. Math. Anal. Appl. 380 (2011) 493-500.

[5] K. Gröchenig and A. Haas, Self-similar lattice tilings, J. Fourier Anal. Appl. 1 (1994), 131-170.

[6] D. Hacon, N.C. Saldanha and J.J.P. Veerman, Remarks on self-affine tilings, Experiment. Math. 3 (1994), 317-327.

[7] M. Hata, On the structure of self-similar sets, Japan J. Appl. Math. 2 (1985), no.2, 381-414.

[8] X.G. He, I. Kirat and K.S. Lau, Height reducing property of polynomials and self-affine tiles, Geom. Dedicata (2011) 152:153-164.

[9] I. Kirat, Disk-like tiles and self-affine curves with non-collinear digits, Math. Comp. 79 (2010), 1019-1045.

[10] I. Kirat and K.S. Lau, On the connectedness of self-affine tiles, J. London Math. Soc. 6227 (2000), 291-304.

[11] I. Kirat, K.S. Lau and H. Rao, Expanding polynomials and connectedness of self-affine tiles, Discrete Comput. Geom. 31 (2004), 275-286.

[12] J.C. Lagarias and Y. Wang, Self-affine tiles in $\mathbb{R}^{n}$, Adv. Math. 121 (1996) 21-49.

[13] K.S. Leung and K.S. Lau, Disk-likeness of planar self-affine tiles, Trans. Amer. Math. Soc. 359 (2007), 3337-3355.

[14] K.S. Leung and J.J. Luo, Connectedness of planar self-affine sets associated with nonconsecutive collinear digit sets, J. Math. Anal. Appl. 395 (2012) 208-217.

Department of Mathematics and Information Technology, The Hong Kong Institute of Education, Hong Kong

E-mail address: ksleung@ied.edu.hk

Department of Mathematics, Shantou Uinversity, Shantou 515063, China

E-mail address: luojun@stu.edu.cn 